\title{
Bifidobacterium bifidum-Mediated Specific Delivery of Nanoparticles for Tumor Therapy
}

\section{Yu Tang \\ Chun Chen \\ Binglei Jiang \\ Lu Wang \\ Fujie Jiang (D) \\ Disen Wang \\ Yaotai Wang \\ Haiyan Yang \\ Xia Ou \\ Yan Du \\ Qi Wang \\ Jianzhong Zou}

State Key Laboratory of Ultrasound in Medicine and Engineering, Chongqing Key Laboratory of Biomedical Engineering, College of Biomedical Engineering,

Chongqing Medical University,

Chongqing, 400016, People's Republic of China
Correspondence: Jianzhong Zou State Key Laboratory of Ultrasound in Medicine and Engineering, Chongqing Key Laboratory of Biomedical Engineering,

College of Biomedical Engineering,

Chongqing Medical University, Chongqing,

4000 16, People's Republic of China

Tel +86-13708302390

Email zoujzh@cqmu.edu.cn
Purpose: Hypoxia is considered to be obstructive to tumor treatment, but the reduced oxygen surroundings provide a suitable habitat for Bifidobacterium bifidum (BF) to colonize. The anaerobe BF selectively colonizes into tumors following systemic injection due to its preference for the hypoxia in the tumor cores. Therefore, BF may be a potential targeting agent which could be used effectively in tumor treatment. We aimed to determine whether a novel BF-mediated strategy, that was designed to deliver AP-PFH/PLGA NPs (aptamers CCFM641-5-functionalized Perfluorohexane (PFH) loaded poly(lactic-co-glycolic acid) (PLGA) nanoparticles) by aptamer-directed approach into solid tumor based on the tumortargeting ability of BF, could improve efficiency of high intensity focused ultrasound (HIFU) treatment of breast cancer.

Methods: We synthesized AP-PFH/PLGA NPs using double emulsion method and carbodiimide method. Then, we evaluated targeting ability of AP-PFH/PLGA NPs to BF in vivo. Finally, we studied the efficacy of HIFU ablation based on BF plus AP-PFH/PLGA NPs (BFmediated HIFU ablation) in tumor.

Results: The elaborately designed AP-PFH/PLGA NPs can target BF colonized in tumor to achieve high tumor accumulation, which can significantly enhance HIFU therapeutic efficiency. We also found that, compared with traditional chemotherapy, this therapy not only inhibits tumor growth, but also significantly prolongs the survival time of mice. More importantly, this treatment strategy has no obvious side effects.

Conclusion: We successfully established a novel therapy method, BF-mediated HIFU ablation, which provides an excellent platform for highly efficient and non-invasive therapy of tumor.

Keywords: high intensity focused ultrasound, aptamers, nanoparticles, Bifidobacterium bifidum, tumor treatment

\section{Introduction}

The treatment of cancer has been a universal conundrum for years. Despite the unprecedented potential of high intensity focused ultrasound (HIFU) for cancer therapy, to achieve precise HIFU ablation has remained a challenge. ${ }^{1-3}$ HIFU destroys the tumor tissue by focusing the ultrasound from an in vitro transducer into tumor tissue to generate a high temperature within a few seconds and then induces obvious thermal coagulation necrosis. ${ }^{4}$ Unfortunately, the efficacy of HIFU ablation is low when the focused ultrasound reaches the deep-seated organs due to the attenuation of ultrasound energy along the ultrasound pathway, which often needs higher ultrasound power and longer ablation duration. The simple increase of ultrasound energy and ablation duration will cause undesired heating in tissues and lead to unknown adverse events to 
normal tissue. ${ }^{5}$ Thus, obtaining higher therapeutic efficacy with lower therapeutic power has remained a challenge in HIFU therapy. The efficient route to enhance the efficacy of HIFU ablation was by introducing synergistic agents to change the acoustic environment of tissues, increase the ultrasonic cavitation effect and enhance the energy deposition. ${ }^{6}$ Typically, microbubbles were used as synergistic agents in HIFU ablation. ${ }^{7-9}$ However, microbubbles have limited contributions to improve the efficiency of HIFU ablation due to the particle size of several micrometers which makes it difficult to penetrate tumor blood vessels and enter the tumor tissue. ${ }^{10}$ Nanoscale bubbles also only have very limited contributions to improve the efficiency of HIFU ablation since the ultrasound responsiveness is decreased significantly. ${ }^{11}$ How to minimize the particle size yet retain the efficacy of HIFU ablation has remained important in HIFU therapy. Fortunately, perfluorohexane (PFH) loaded poly(lactic-co-glycolic acid) (PLGA) nanoparticles (PFH/PLGA NPs) could efficiently solve this critical issue. ${ }^{7,11}$ PLGA is a favored substance for the fabrication of nanoparticles (NPs) due to its specific features, such as favorable biosafety and easy chemical modifications and is approved by the United States Food and Drug Administration (FDA). ${ }^{12,13} \mathrm{PFH}$ also is a typical highly biocompatible fluorocarbon with boiling point at $56^{\circ} \mathrm{C} .{ }^{14}$ PFH/PLGA NPs are special "small-to-big" phasetransformation nanoparticles, which could be in situ transformed into large microbubbles because of the temperatureresponsive liquid-gas phase transition of PFH by HIFU triggering, and this further significantly enhances the HIFU ablation efficacy by changing the acoustic environment and enhancing the deposition of ultrasound energy. The particle size of these PFH/PLGA NPs is a nanometer, making it easier to accumulate in the tumor. ${ }^{10,15-17}$

To achieve precise HIFU ablation, the boundaries between tumor and normal tissue should be distinguished. However, most synergistic agents are stymied by non-specific organ biodistribution. Therefore, it is a great challenge to improve the targeting efficiency of synergistic agents. However, the traditional targeting delivery of synergistic agents is based on the marker molecules over-expressed by tumor cells. ${ }^{18}$ Because of the heterogeneity of tumor, some tumor cells may not express or have low expression of the corresponding marker molecules, so the efficiency of synergistic agents' delivery is not high. Tumor heterogeneity is also a key factor leading to the failure of tumor targeted therapy. ${ }^{19,20}$

Research shows that some species of anaerobic bacteria, such as Bifidobacterium, can selectively germinate and grow in the hypoxic regions of solid tumors. There is no proliferation in healthy tissues rich in oxygen. Thus, Bifidobacterium may be a marker which would aid in distinguishing tumor and normal tissues and could be used effectively in tumor diagnosis or treatment. ${ }^{21-24}$ Therefore, we sought to harness the tumor-targeting ability of BF to deliver AP-PFH/PLGA NPs into the tumor for highly efficient HIFU therapy of cancer.

To achieve the effective delivery, some antibodies, peptides or aptamers have to conjugate with NPs to prepare targeting nanoparticles. Aptamers are singlestranded DNA or RNA typically screened through Systematic Evolution of Ligands by Exponential Enrichment (SELEX). Aptamers have unique tertiary structures and can specifically bind with molecular targets by a combination of non-covalent interactions, such as van der Waals forces, hydrogen bonding, and stacking interactions. ${ }^{25}$ Aptamers have some advantages, such as ease of chemical synthesis, small size, low molecular weight and lack of immunogenicity compared with antibodies. These features make aptamers a good candidate for conjugation to nanoparticles for targeted imaging, diagnostic and therapeutic purposes. ${ }^{26-28}$ Therefore, in this study, specific delivery approaches, an aptamerdirected method, were designed to deliver AP-PFH /PLGA NPs into solid tumor based on the tumortargeting ability of BF for HIFU ablation.

In our study, the aptamers CCFM641-5-functionalized PFH/PLGA NPs (AP-PFH/PLGA NPs) can target Bifidobacterium bifidum (BF) colonized in tumor to achieve high tumor accumulation, which were further used to enhance HIFU therapeutic efficiency of solid tumors. HIFU ablation based on BF plus AP-PFH/PLGA NPs (BF-mediated HIFU ablation) can significantly inhibit tumor growth and prolong the survival period of mice bearing breast cancer xenografts, compared with traditional chemotherapy. Notably, this work is not purely limited to construct a new targeting synergistic agent but pave a direction to BF-mediated HIFU ablation using the anaerobic bacterial approach for tumor therapy.

\section{Materials and Methods Materials}

Polyethylene glycol (PEG), poly(lactic-co-glycolic acid) (PLGA) (50:50, MW=15000) and PLGA-PEG-COOH was purchased from Daigang BIO Engineer Ltd., Co. (Shan Dong, China). Chloroform $\left(\mathrm{CHCl}_{3}\right)$ and isopropyl alcohol 
were purchased from Chongqing East Chemical Industry Ltd., Co. (Chongqing, China). Poly(vinyl alcohol) (PVA, $\mathrm{MW}=25000$ ) were purchased from Sigma-Aldrich Chemical Co. (St Louis, MO, USA). Fluorescein isothiocyanate (FITC) were purchased from BioFroxx Ltd., Co. (Germany). 1,1-dioctadecyl-3,3,3,3-tetramethylindotricarbocyanine iodide (DiR) was purchased from AAT Bioquest Inc. (USA). 4,6-diamidino-2-phenylindole (DAPI) and 1,1'dioctadecyl-3,3,3',3'-tetramethylindocarbocyanine perchlorate (DiI) were purchased from Beyotime Biotechnology Ltd., Co. (Shanghai, China). 1-ethyl-3- (3-dimethylaminopropyl) carbodiimide hydrochloride (EDC), N-hydroxysulfosuccinimide (sulfo-NHS), MES buffer and PFH were purchased from Aladdin Co., Ltd. (Shanghai, China). Aptamer CCFM641-5 ${ }^{29}$ (sequence: 5'AGCAGCACAGAGGTCAGATGTGCGTGAGCGGTAGCCCCGTACGACCCACTGTGGTTGGGCCCTATGCGTGCTACCGTGAA-3', 80 bp), 5'-NH2-modified CCFM6415 aptamer and 5'-FAM-modified CCFM641-5 aptamer were synthesized by Sangon Biotech Co., Ltd (Shanghai, China). Cell Counting Kit-8 (CCK-8) assay was purchased from Dojindo Laboratories (Kumamoto, Japan).

\section{Methods}

Growth of Bifidobacterium bifidum Strain ATCC2952I Bifidobacterium bifidum strain ATCC29521 (BF, American Type Culture Collection) was anaerobically cultured on de Man-Rogosa-Sharpe (MRS) broth supplied with $0.05 \%$ L-cysteine hydrochloride monohydrate at $37^{\circ} \mathrm{C}$ to early-log phase. $\mathrm{BF}$ were collected by centrifugation at $4000 \mathrm{rpm}$ for $10 \mathrm{~min}$ at $4^{\circ} \mathrm{C}$. BF concentration was calculated by serially diluting the $\mathrm{BF}$ suspension, anaerobically culturing the dilutions on solid $\mathrm{LB}$ agar at $37^{\circ} \mathrm{C}$ for $72 \mathrm{~h}$, and counting colony forming units (CFUs). BF were resuspended with icecold sterile PBS to adjust the concentration of $10^{8} \mathrm{CFU} /$ $\mathrm{mL}$ for further use.

\section{Cell Culture}

The human breast cancer MDA-MB-231 cells and human umbilical vein endothelial cells (HUVECs) were obtained from Chinese Academy of Sciences Cell Bank. All cells were cultured in Roswell Park Memorial Institute-1640 (RPMI-1640) medium containing 1\% penicillin/streptomycin and 10\% fetal bovine serum (FBS) and incubated under $5 \% \mathrm{CO}_{2}$ atmosphere at $37^{\circ} \mathrm{C}$.

\section{Animal Model}

All female BALB/c nude mice (6 8 weeks old) were purchased from the Experimental Animal Center of Chongqing Medical University and housed in a constant temperature and humidity condition. For the establishment of the MDA-MB-231 xenografts nude mice models, female $\mathrm{BALB} / \mathrm{c}$ nude mice were respectively subcutaneously injected in the right flank with $1 \times 10^{6}$ MDA-MB -231 cells suspended in $100 \mu \mathrm{L}$ phosphate-buffered saline (PBS). Generally, tumors were palpable within 14 days. Tumor volume was calculated as $\left(\right.$ length $\times$ width $\left.^{2}\right) / 2$. All the experimental procedures were approved by the animal ethics committee of Chongqing Medical University. All procedures involving animals were conducted with the guidelines of the Institutional Animal Care and Use Committee of Chongqing Medical University.

\section{In vivo Biodistribution of $\mathrm{BF}$}

To study the biodistribution of BF, when the tumor volume reached approximately $100-250 \mathrm{~mm}^{3}, 15$ nude mice bearing MDA-MB-231 xenografts were intravenously injected with $0.2 \mathrm{~mL}$ PBS containing $10^{7} \mathrm{CFU}$ of BF once every 24 h, three times in total. At 1 h, 24 h, 48 h, 120 h and 168 $\mathrm{h}$ after the third injection, mice were sacrificed (three per time). Then their heart, liver, spleen, lung, kidney and tumor were collected and weighed. Each tissue sample was homogenized in PBS. The amount of BF in each tissue was determined by serially diluting tissue homogenates, anaerobically culturing the dilutions on solid LB agar at $37^{\circ} \mathrm{C}$ for $72 \mathrm{~h}$ and counting CFU.

\section{In vivo Biosafety of $B F$}

Experimental details can be found in the supporting information.

\section{Preparation of PFH/ PLGA NPs}

PFH/PLGA NPs were prepared by a double emulsion method. $^{30,31}$ First, $100 \mathrm{mg}$ PLGA-PEG-COOH was added to the organic phase of $2 \mathrm{~mL} \mathrm{CHCl}_{3}$ and then $0.2 \mathrm{~mL}$ PFH was added. In an ice bath, the mixture was emulsified by an ultrasonic cell crusher (Sonics \& Materials, Inc., USA) with power of $105 \mathrm{~W}$ for $1 \mathrm{~min}$. Subsequently, $4 \mathrm{~mL}$ cold PVA solution (4\%, w/v) was added into this initial emulsion. The mixture was emulsified again by the ultrasonic cell crusher with power of $105 \mathrm{~W}$ for $2 \mathrm{~min} 30 \mathrm{~s}$ to obtain the second emulsion. $8 \mathrm{~mL} 2 \%$ isopropyl alcohol solution was poured into the second emulsion, and then the prepared emulsion was mechanically stirred in an ice bath for 3-6 $\mathrm{h}$ to 
extract $\mathrm{CHCl}_{3}$. After the prepared emulsion was centrifuged at $9000 \mathrm{rpm}$ for $5 \mathrm{~min}$, the supernatant was discarded and the precipitate was washed by deionized water. The process of centrifugation and washing were repeated three times. Finally, the precipitate was mixed with $2 \mathrm{~mL}$ deionized water and lyophilized for $48 \mathrm{~h}$. The dried samples were stored at $-20^{\circ} \mathrm{C}$ for use. To prepare fluorescent PFH/PLGA NPs, DiI or DiR was added to the $\mathrm{CHCl}_{3}$ solution in the dark. The rest of the steps are the same.

\section{Conjugation of AP to PFH/PLGA NPs}

AP was conjugated to the surface of PFH/PLGA NPs according to a carbodiimide method. The prepared PFH/ PLGA NPs $(10 \mathrm{mg})$ was dissolved in $1 \mathrm{~mL}$ MES buffer $(0.1 \mathrm{~mol} / \mathrm{L}, \mathrm{pH}=5.5)$. Then $4 \mathrm{mg}$ EDC and $11 \mathrm{mg}$ sulfoNHS was added to the suspension and incubated for 30 min at room temperature. ${ }^{32-34}$ After solution was centrifuged at $9000 \mathrm{rpm}$ for $5 \mathrm{~min}$, the supernatant was discarded and the precipitate was washed by deionized water to remove excess EDC and sulfo-NHS. The process of centrifugation and washing were repeated three times. The precipitate and an appropriate amount of $5^{\prime}-\mathrm{NH}_{2}-$ modified AP (1 ug/ $\mu \mathrm{L})$ was dispersed into MES buffer $(0.1 \mathrm{~mol} / \mathrm{L}, \mathrm{PH}=8.0)$ and incubated for $12 \mathrm{~h}$ at room temperature. The prepared aptamer-NPs conjugates were washed and resuspended with deionized water. The samples were stored at $-4^{\circ} \mathrm{C}$ for use. To prepare fluorescent AP-PFH/PLGA NPs, PFH/PLGA NPs was replaced with DiI-labeled PFH/PLGA NPs or DiR-labeled PFH/PLGA NPs. The rest of the steps are the same. The AP-PFH /PLGA NPs concentration " $\mathrm{mg} / \mathrm{mL}$ " is calculated by dividing the weight of AP-PFH/PLGA NPs by the volume of the solution.

\section{Assessment of Binding Between AP and PFH/PLGA NPs}

AP labeled with FAM was conjugated to PFH/PLGA NPs as described previously. Flow cytometry (FCM, BD FACSVantage SE, USA) was used to determine the binding efficiency between FAM-labeled AP and PFH/ PLGA NPs.

\section{Characterization of AP-PFH/PLGA NPs}

The scanning electron microscopy (SEM, Hitachi S-3400N, Japan) and transmission electron microscopy (TEM, Hitachi H-7600, Japan) were used to evaluate the morphological characteristics of AP-PFH/PLGA NPs. The mean particle sizes and zeta potential of different NPs were measured using a Malvern Zetasizer Nano ZS instrument (Malvern Instruments, UK). Then, the mean particle sizes of AP-PFH/PLGA NPs dissolved in PBS were measured with prolonged time duration $(1,2,3,4,5,6$ and 7 days) and the AP-PFH/PLGA NPs $(15 \mathrm{mg} / \mathrm{mL})$ were also observed by digital photographs.

\section{Toxicity Evaluation of AP-PFH/PLGA NPs}

Experimental details can be found in the supporting information.

\section{Targeting Binding Between BF and AP-PFH/PLGA NPs}

In order to detect the binding between $\mathrm{BF}$ and AP-PFH /PLGA NPs in vitro, DiI-PFH/PLGA NPs and DiI-APPFH/PLGA NPs was prepared as previously described. $\mathrm{BF}$ was harvested by centrifugation at $4000 \mathrm{rpm}$ for 10 $\min$ at $4^{\circ} \mathrm{C}$. The concentration was adjusted to $10^{8} \mathrm{CFU} /$ $\mathrm{mL}$. After staining by FITC, $0.2 \mathrm{~mL}$ BF was divided into 2 test tubes $(\mathrm{BF}+\mathrm{PFH} / \mathrm{PLGA}$ group and $\mathrm{BF}+\mathrm{AP}-\mathrm{PFH} / \mathrm{PLGA}$ group). For $\mathrm{BF}+\mathrm{PFH} / \mathrm{PLGA}$ group, $0.5 \mathrm{~mL}$ DiI-PFH /PLGA NPs $(10 \mathrm{mg} / \mathrm{mL})$ was added. For BF+AP-PFH /PLGA group, $0.5 \mathrm{~mL}$ DiI-AP-PFH/PLGA NPs $(10 \mathrm{mg} /$ $\mathrm{mL})$ was added. The mixed solution in each group was reacted at room temperature for $2 \mathrm{~h}$. The connection between BF and NPs was observed under the confocal laser scanning microscope (CLSM, LeicaSP8, Germany). Similar to the methods for the CLSM test, FCM was also carried out to examine the binding efficiency between BF (without staining by FITC) and DiI-labeled NPs.

In order to detect the binding between $\mathrm{BF}$ and $\mathrm{AP}-\mathrm{PFH}$ /PLGA NPs in vivo, when the tumor volume reached approximately $100-250 \mathrm{~mm}^{3}$ after 14 days of inoculation, 9 nude mice bearing MDA-MB-231 xenografts were randomly divided into three groups with 3 mice in each group (AP-PFH/PLGA group, BF+PFH/PLGA group and BF + AP-PFH/PLGA group). The mice in AP-PFH/PLGA group, $\mathrm{BF}+\mathrm{PFH} / \mathrm{PLGA}$ group and $\mathrm{BF}+\mathrm{AP}-\mathrm{PFH} / \mathrm{PLGA}$ group were respectively intravenously injected with $0.2 \mathrm{~mL}$ PBS, PBS containing $10^{7} \mathrm{CFU}$ of BF and PBS containing $10^{7} \mathrm{CFU}$ of BF once every $24 \mathrm{~h}$, three times in total. On $7 \mathrm{~d}$ after the third injection, the mice in the AP$\mathrm{PFH} / \mathrm{PLGA}$ group, $\mathrm{BF}+\mathrm{PFH} / \mathrm{PLGA}$ group and $\mathrm{BF}+\mathrm{AP}-$ PFH/PLGA group were respectively intravenously injected with $0.4 \mathrm{~mL}$ DiR-AP-PFH/PLGA NPs $(10 \mathrm{mg} /$ $\mathrm{mL})$, DiR-PFH/PLGA NPs $(10 \mathrm{mg} / \mathrm{mL})$, DiR-AP-PFH /PLGA NPs $(10 \mathrm{mg} / \mathrm{mL})$. Images were taken at different time points (before injection, on $1 \mathrm{~h}, 8 \mathrm{~h}, 24 \mathrm{~h}$ and 48 
$\mathrm{h}$ after injection NPs) using the LB983 in vivo imaging system (Berthold Technologies $\mathrm{GmbH} \&$ Co. KG, Germany). All nude mice in the three groups were euthanized on $48 \mathrm{~h}$ after injection with NPs. Then tumors and major organs were harvested for immediate observation of fluorescence.

In addition, 36 nude mice bearing MDA-MB-231 xenografts were randomly divided into three groups with 12 mice in each group (AP-PFH/PLGA group, $\mathrm{BF}+\mathrm{PFH} /$ PLGA group and $\mathrm{BF}+\mathrm{AP}-\mathrm{PFH} / \mathrm{PLGA}$ group) and received the same treatment as those described above except that the NPs were labeled with DiI. Three mice in each group were respectively euthanized at $1 \mathrm{~h}, 8 \mathrm{~h}, 24 \mathrm{~h}$ and 48 $\mathrm{h}$ after injection. Then, tumors were immediately harvested for ultrathin sections and then the sections were stained with DAPI for confocal laser scanning microscope (CLSM, A1R; Nikon, Tokyo, Japan) detection.

\section{Evaluation of Synergistic Effect of AP-PFH/PLGA NPs for BF-Mediated HIFU Ablation}

A Model-JC200 Focused Ultrasound Tumor Therapeutic system (Chongqing Haifu Medical Technology Co., Ltd., Chongqing, China) was used for all HIFU experiments. The focal length, diameter, and operating frequency used in this study were $220 \mathrm{~mm}, 145 \mathrm{~mm}$, and $0.94 \mathrm{MHz}$, respectively. Two units, a therapeutic ultrasound unit and a diagnostic ultrasound unit, compose this device. A highenergy ultrasound beam was emitted from the therapeutic transducer to damage the interesting tissue, and the ultrasound grayscale changes in the targeted tissue were monitored using an ultrasound diagnostic transducer (Esaote, Italy, with a probe frequency range of 3.5-5.0 MHz) and analyzed by Gray Val 1.0 software provided with the therapeutic system.

When the tumor volume reached approximately 400$600 \mathrm{~mm}^{3}$, 40 nude mice bearing MDA-MB-231 xenografts were randomly divided into four groups (PBS group, AP-PFH/PLGA group, BF+PFH/PLGA group, $\mathrm{BF}+\mathrm{AP}-\mathrm{PFH} / \mathrm{PLGA}$ group) with 10 mice in each group. Mice in PBS group and AP-PFH/PLGA group were intravenously injected with $0.2 \mathrm{~mL}$ PBS once every $24 \mathrm{~h}$, three times in total. Mice in $\mathrm{BF}+\mathrm{PFH} /$ PLGA group and BF+AP-PFH/PLGA group were injected with $0.2 \mathrm{~mL}$ PBS containing $10^{7} \mathrm{CFU}$ of $\mathrm{BF}$ once every $24 \mathrm{~h}$, three times in total. On day 7 after the third injection, $0.4 \mathrm{~mL}$ PBS, AP-PFH/PLGA $(10 \mathrm{mg} /$ $\mathrm{mL}), \mathrm{PFH} / \mathrm{PLGA}(10 \mathrm{mg} / \mathrm{mL})$ and AP-PFH/PLGA NPs $(10 \mathrm{mg} / \mathrm{mL})$ was respectively injected into the mice in
PBS group, AP-PFH/PLGA group, BF+PFH/PLGA group and $\mathrm{BF}+\mathrm{AP}-\mathrm{PFH} / \mathrm{PLGA}$ group. At $24 \mathrm{~h}$ after injection, mice in the four groups were anesthetized and placed on the HIFU treatment bed in a prone position. The tumor site was completely immersed in degassed water. All mice were treated with the same power in the HIFU ablation experiments (acoustic power $120 \mathrm{~W}$, irradiation duration $3 \mathrm{~s}$ ). Before and after ablation, gray-scale of the targeted zone was automatically compared using Gray Val 1.0 software affiliated to the HIFU device. On $24 \mathrm{~h}$ after HIFU ablation, 5 mice in each group were randomly sacrificed and the tumors were harvested. The tumor was completely dissected and cut longitudinally from the middle in the direction of HIFU ablation. Tumor tissue was stained with 2,3,5-triphenyltetrazolium chloride (TTC) solution at $37^{\circ} \mathrm{C}$ for $30 \mathrm{~min}$ to determine the coagulation necrosis volume. The coagulation necrosis volume (V) was calculated as follows: $\mathrm{V}\left(\mathrm{mm}^{3}\right)=(\pi / 6) \times$ length $\times$ width $\times$ depth. The remaining 5 mice in each group were sacrificed and the tumors were harvested and fixed in $4 \%$ polyoxymethylene routinely processed, paraffin embedded and sectioned. H\&E staining on tissues sections was used for histopathological analysis. To evaluate apoptosis, Terminal-deoxynucleotidyl Transferase Mediated Nick End Labeling (TUNEL assay) method was performed on these tissue sections. The apoptotic index (AI) was calculated as the ratio of positively stained cells to all cells, which were obtained from at least 5 randomly selected fields $(\times 400$ magnification) by blinded observers.

\section{In vivo Anti-Tumor Effect of BF-Mediated HIFU Ablation}

When the tumor volume reached approximately 100$250 \mathrm{~mm}^{3}$ after 14 days of inoculation, 15 nude mice bearing MDA-MB-231 xenografts were randomly divided into three groups with 5 mice in each group (PBS group, docetaxel group, $\mathrm{HIFU}+\mathrm{BF}+\mathrm{AP}-\mathrm{PFH}$ /PLGA group). Mice were euthanized once tumor volume approached burden as defined by IACUC. Mice were euthanized at day 70 after tumor inoculation, if they had not yet been euthanized. On day 0 , mice in PBS group were injected with PBS once per week and mice in docetaxel group were injected with free docetaxel solution $(10 \mathrm{mg} / \mathrm{mL}$ of docetaxel concentration in PBS containing $25 \% \mathrm{w} / \mathrm{v}$ tween 80 and $9.75 \% \mathrm{v} / \mathrm{v}$ ethanol) at a dose of $10 \mathrm{mg} / \mathrm{kg}$ once per week. ${ }^{35-38}$ Mice in HIFU + 
$\mathrm{BF}+\mathrm{AP}-\mathrm{PFH} / \mathrm{PLGA}$ group were injected with $0.2 \mathrm{~mL}$ PBS containing $10^{7} \mathrm{CFU}$ of BF once every $24 \mathrm{~h}$, three times in total, and on day 7 after the third injection, $0.4 \mathrm{~mL}$ AP-PFH/PLGA NPs $(10 \mathrm{mg} / \mathrm{mL})$ was injected. Then, $24 \mathrm{~h}$ after injection, mice were anesthetized and placed on the HIFU treatment bed in a prone position. Using point irradiation, intermittent emission therapy, the treatment is layer by layer from the deepest to the shallowest part of the emission source (acoustic power 120 $\mathrm{W}$, irradiation duration $3 \mathrm{~s}$ ). Treatment duration is 6-9 s. Tumor volumes and body weights of the mice were recorded every 3 days. Digital photographs of the mice were taken every 5 days for 20 days after the treatment.

\section{Statistical Analysis}

All data were analyzed with GraphPad Prism 7.0 version. Quantitative data are expressed as the mean \pm standard deviation. Student's $t$-test, one-way analysis of variance (ANOVA), two-way ANOVA and Log-rank (Mantel-Cox) test were used for data analysis. $\mathrm{P}$ values $<0.05$ were considered statistically significant, $* \mathrm{P}<0.05, * * \mathrm{P}<0.01, * * * \mathrm{P}<0.001$.

\section{Results and Discussion} AP-PFH/PLGA NPs for BF-Mediated

\section{HIFU Ablation}

Herein, as shown in Scheme 1, the BF-mediated strategy was designed to deliver AP-PFH/PLGA NPs by aptamerdirected approach into solid tumors based on the tumortargeting ability of BF to enhance the treatment efficiency of HIFU: the injection of BF first, followed by the administration of the aptamer-NPs conjugates to specifically target BF colonized in tumor. Specifically, in the first step, BF was injected into the nude mice bearing MDAMB-231 xenografts through the tail vein once every $24 \mathrm{~h}$, three times in total. The aptamer CCFM641-5 (AP) is an aptamer specifically for BF and was used to guide targeting binding between BF and AP-PFH/PLGA NPs in the

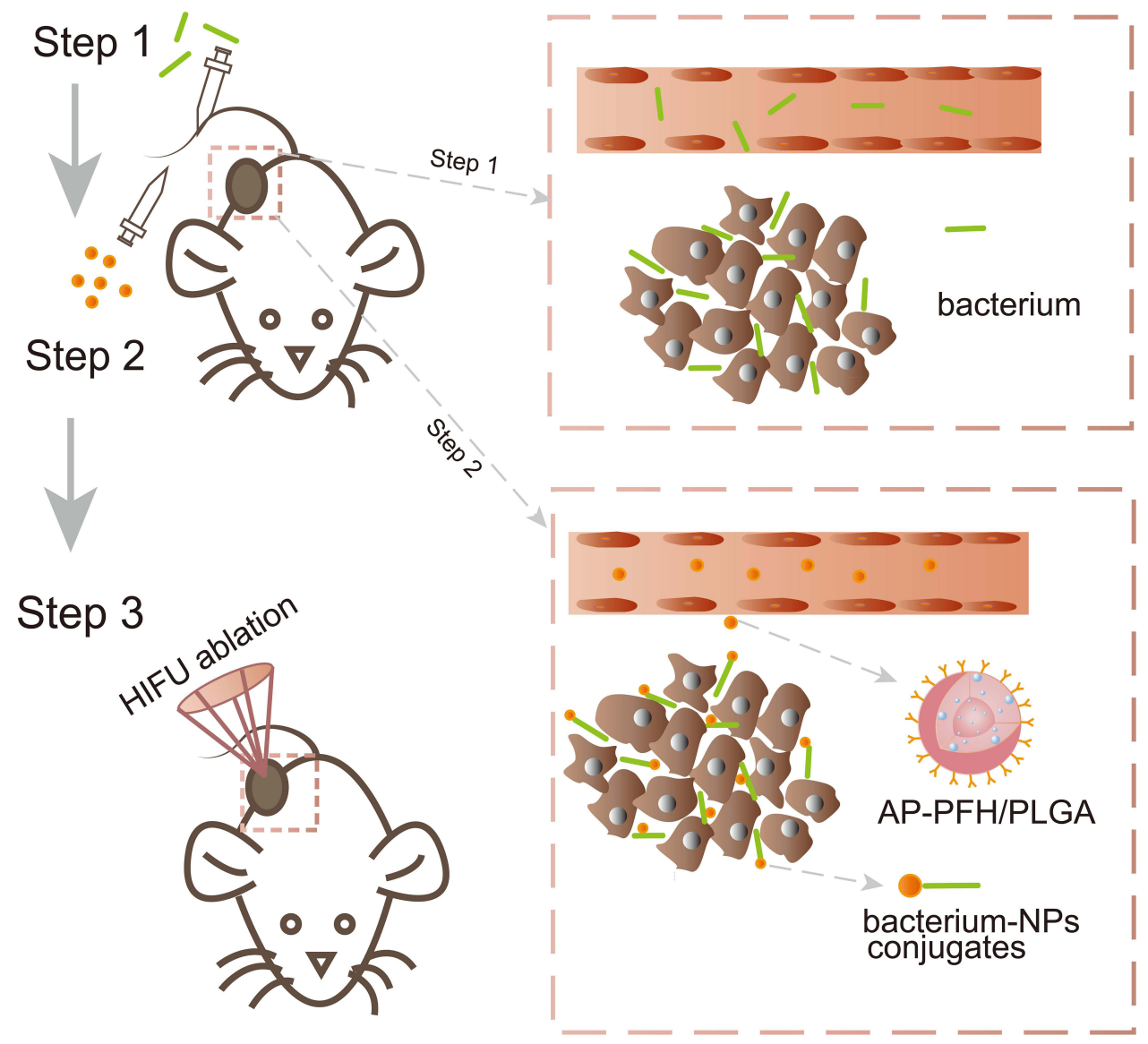

Scheme I Schematic illustration of targeting AP-PFH/PLGA NPs for BF-mediated HIFU ablation.

Abbreviations: PFH, Perfluorohexane; PLGA, poly(lactic-co-glycolic acid); NPs, nanoparticles; AP, aptamers CCFM64I-5; BF, Bifidobacterium bifidum strain ATCC2952I; HIFU, High intensity focused ultrasound. 
tumor area. In the second step, on day 7 after the third injection, AP-PFH/PLGA NPs were injected. Guided by aptamers, AP-PFH/PLGA NPs can target BF in tumor. In the third step, when a large amount of NPs accumulate in the tumor $24 \mathrm{~h}$ after injection, HIFU ablation is used for tumor treatment.

\section{Tumor-Targeting Ability and Biosafety Evaluation of $\mathrm{BF}$}

To determine whether BF could selectively germinate and grow in tumors in vivo, nude mice bearing MDA-MB-231 xenografts were intravenously injected with BF once every $24 \mathrm{~h}$, three times in total. At $1 \mathrm{~h}, 24 \mathrm{~h}, 48 \mathrm{~h}, 120 \mathrm{~h}$ and 168 $\mathrm{h}$ after the third injection, hearts, livers, spleens, lungs, kidneys, and tumors were collected. BF in these tissues was quantified by serial dilution homogenates and then counted on solid LB agar plates. As shown in Figure 1A, at $1 \mathrm{~h}$ after the third injection, sporadic colonies grew from tumor homogenates and dense colonies grew from homogenates of major organs. However, at $168 \mathrm{~h}$ after the third injection, almost no colonies grew from homogenates of major organs and dense colonies grew from tumor homogenates. By counting the CFU in each tissue (Figure 1B), it was found that the amount of $\mathrm{BF}$ in the tumor tissue continued to increase for $168 \mathrm{~h}$ after the third injection. Contrastingly, the amount of BF in normal tissues, such as the heart, liver, spleen, lung and kidney, began to decrease immediately after injection and BF was almost undetectable at $168 \mathrm{~h}$ after the third injection. These results indicated that BF can survive and proliferates selectively in tumor tissues, but cannot in normal tissues. This phenomenon may be attributed to the unique hypoxic microenvironment of solid tumors and the anaerobic instincts of BF. Abnormal growth of the vasculature of a solid tumor usually creates a hypoxic area in the tumor. ${ }^{39}$ The hypoxic regions of solid tumors with a significant proportion of readings below $2.5 \mathrm{mmHg},{ }^{40}$ may provide $\mathrm{BF}$ a suitable environment to germinate and grow. ${ }^{21-24}$ On the contrary, the average partial pressure of oxygen in normal tissues is 24-66 mm Hg, ${ }^{40}$ which may prevent $\mathrm{BF}$ growing.

We also examined the toxicity of BF. First, the red blood cell (RBC), white blood cell (WBC), platelet (PLT), hemoglobin (HGB), mean corpuscular hemoglobin $(\mathrm{MCH})$, mean corpuscular volume $(\mathrm{MCV})$, alanine aminotransferase (ALT), aspartate aminotransferase (AST), creatinine (CREA) and blood urea nitrogen (BUN) were estimated on days 3, 7, 14 and 28 after the third injection of PBS or BF. The result of routine blood test and blood biochemical analyses in BF group showed no apparent difference compared with PBS group (Figure S1A). Next, H\&E staining (Figure S1B) of the major organs (heart, liver, spleen, lung and kidney) indicated that $\mathrm{BF}$ did not damage the major organs. Together, these results indicate that $\mathrm{BF}$ have excellent tumor-targeting ability and biosafety. Therefore, BF is a potential targeting agent which could be used effectively in tumor treatment.

\section{Synthesis and Characterization of AP-PFH/PLGA NPs}

In order to synthesize a synergistic agent to target $\mathrm{BF}$, PFH/PLGA NPs was constructed by the double emulsion method, and then the AP specific for BF was further coupled to the surface of the PFH/PLGA NPs by carbodiimide method (Figure 2A). First, FCM was applied to confirm the binding efficiency between AP and $\mathrm{PFH} /$ PLGA NPs. As shown in Figure 2B and C, the binding efficiency between AP and PFH/PLGA NPs is $84.9 \% \pm$ $11.1 \%$. Therefore, we successfully prepared AP-PFH /PLGA NPs. Subsequently, we characterized the NPs. The high dispersity, well-defined spherical morphology and homogeneous size of the AP-PFH/PLGA NPs was revealed by SEM (Figure 2D). In TEM image of PFH/ PLGA NPs (Figure S2A) and AP-PFH/PLGA NPs (Figure 2E), dark in the core can be directly observed, indicating that the PFH are successfully encapsulated into PLGA. The particle sizes of PFH/PLGA NPs and APPFH/PLGA NPs were $216.3 \pm 19.4 \mathrm{~nm}$ and $232.4 \pm 25.6$ $\mathrm{nm}$ (Figure 2F), showing a relatively narrow size distribution. In addition, the average zeta potentials of the APPFH/PLGA NPs and PFH/PLGA NPs were $-20.0 \pm 3.13$ $\mathrm{mV}$ and $-17.3 \pm 5.49 \mathrm{mV}$ (Figure S2B). The grafting of AP on the surface of polymer shells exerted no obvious effect on the particle size of AP-PFH/PLGA NPs. Because its particle size is between $200-300 \mathrm{~nm}$ and $<700 \mathrm{~nm}$, this guarantees NPs can penetrate tumor blood vessels and enter the tumor tissue. ${ }^{10,41}$ In addition, PEGylation of the NPs can reduce the rapid elimination by the mononuclear phagocytic system and increase the accumulation by prolonging the circulation time of the NPs. ${ }^{12}$ Meanwhile, within 7 days the mean particle size of AP-PFH/PLGA NPs did not appreciably change when dissolved in PBS, and the particle suspension did not aggregate or precipitate (Figure 2G), revealing the 
A
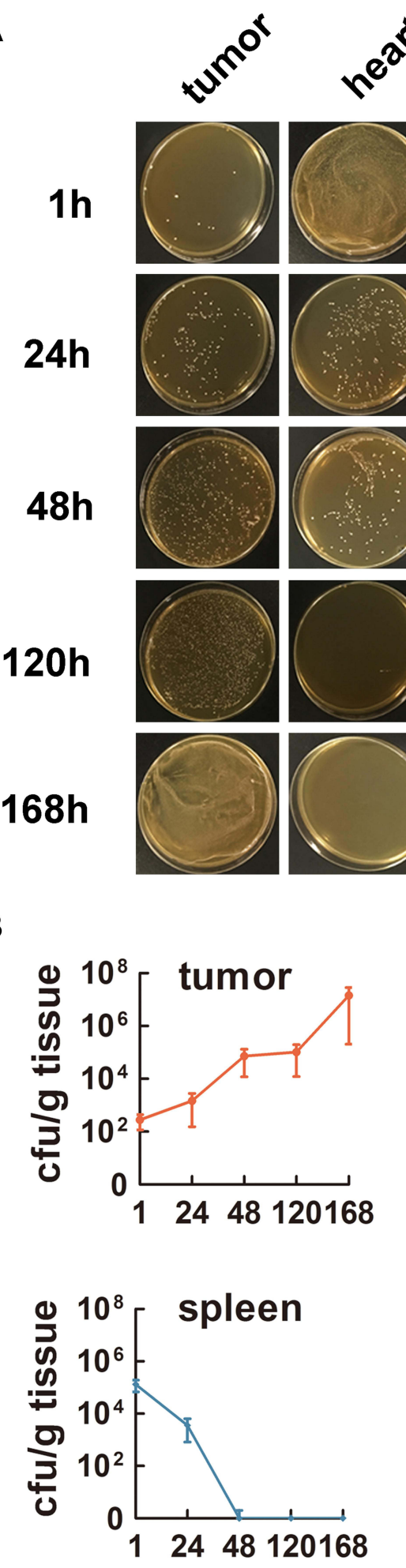
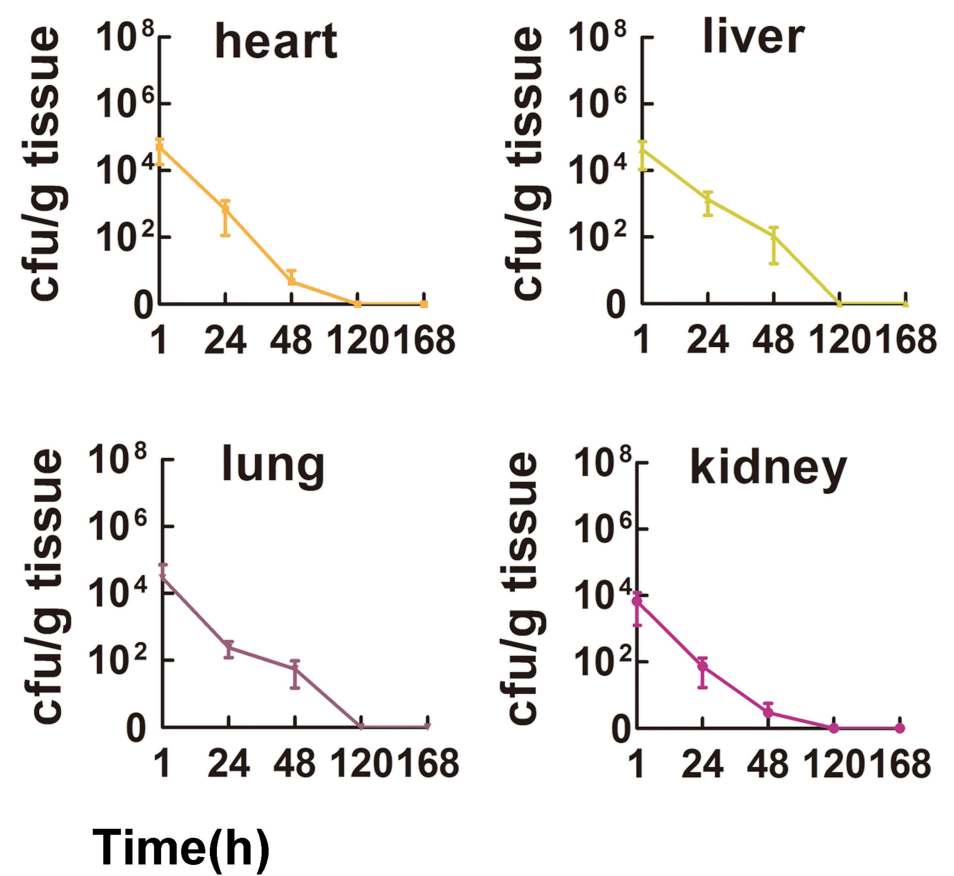

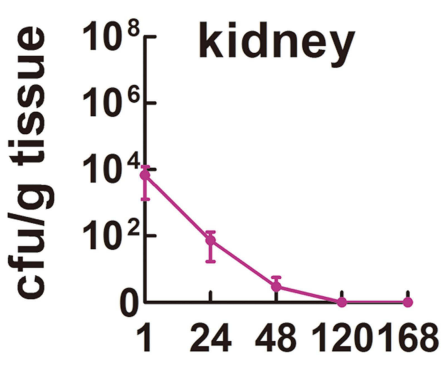

Figure I Tumor-Targeting Ability of BF. (A) Homogenates of tumor tissues and the five organs on different time points after the third injection of BF were cultured on solid $\mathrm{LB}$ agar at $37^{\circ} \mathrm{C}$. (B) Quantification of BF per gram of tumor tissues and the five organs at different time points after the third injection of $B F(n=3)$. Abbreviation: BF, Bifidobacterium bifidum strain ATCC2952I. 
A

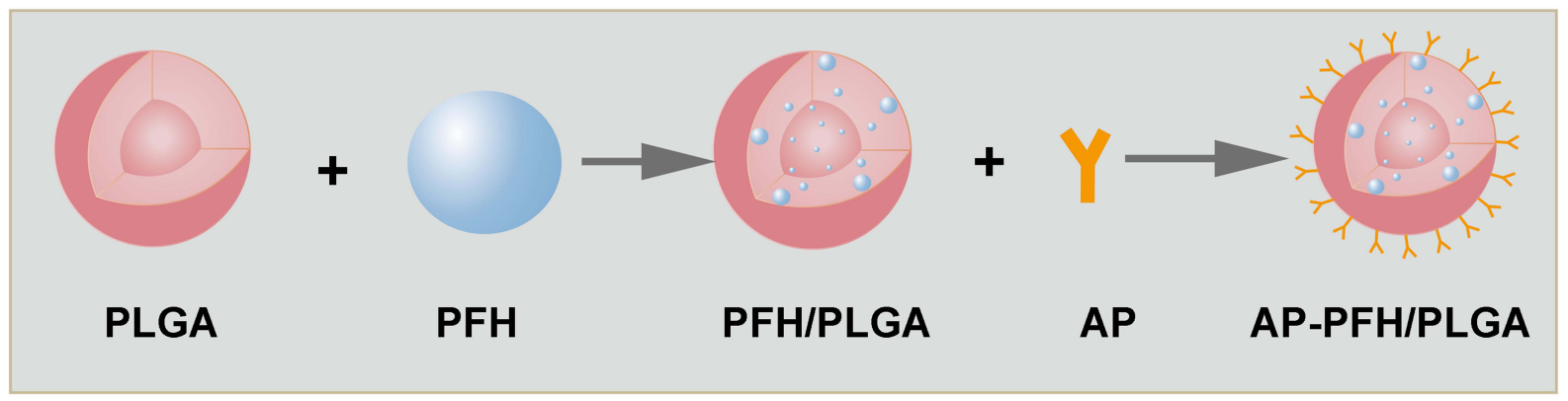

B

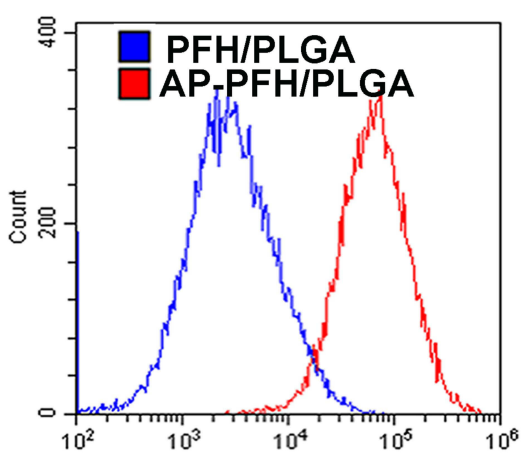

E

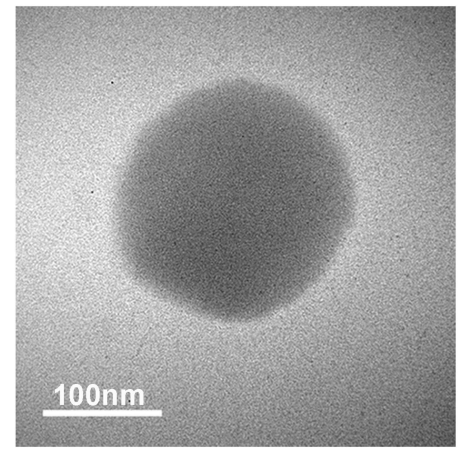

C

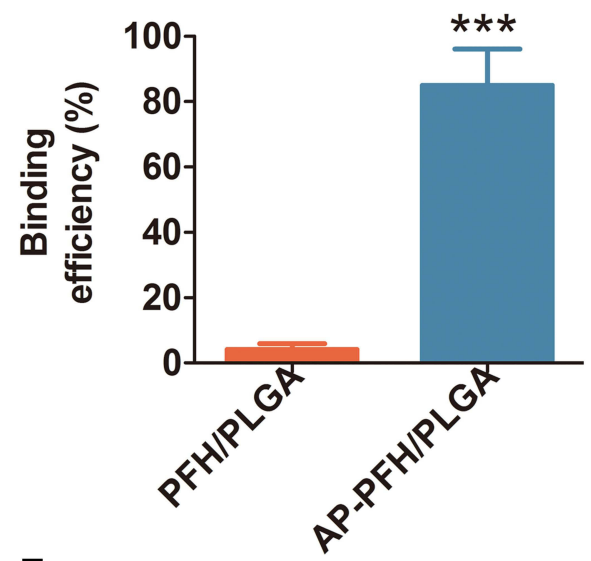

$\mathbf{F}$

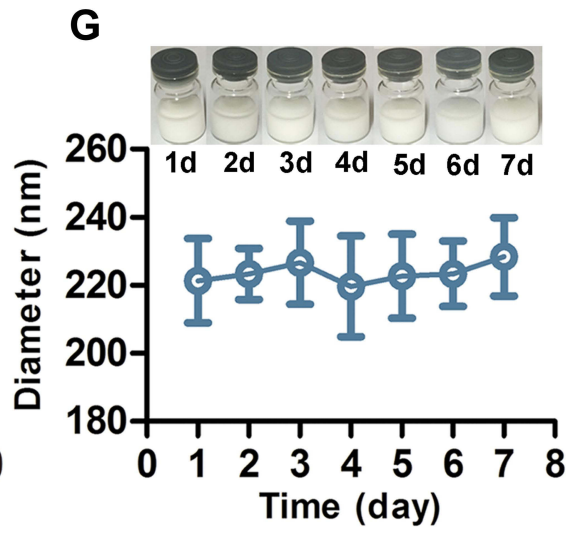

Figure 2 Characterization of AP-PFH/PLGA NPs. (A) The schematic diagram for the synthesis of AP-PFH/PLGA NPs. (B) Flow cytometry analysis binding between AP and PFH/PLGA NPs. (C) Binding efficiency between AP and PFH/PLGA NPs $(n=3$, ***P $<0.00 I)$. (D) SEM image and (E) TEM image of AP-PFH/PLGA NPs. (F) Size distribution of PFH/PLGA NPs and AP-PFH/PLGA NPs. (G) The size distribution of AP-PFH/PLGA NPs in PBS with prolonged time duration ( $n=3$ ); Inset: digital photos of the AP-PFH /PLGA NPs dispersed in PBS ( $15 \mathrm{mg} / \mathrm{mL})$.

Abbreviations: PFH, perfluorohexane; PLGA, poly(lactic-co-glycolic acid); NPs, nanoparticles; AP, aptamers CCFM64I-5; SEM, scanning electron microscopy; TEM, transmission electron microscopy.

excellent long-term stability of the AP-PFH/PLGA NPs for further utilization.

\section{In vitro and in vivo Toxicity of AP-PFH/ PLGA NPs}

To evaluate cytotoxicity of AP-PFH/PLGA NPs, MDA-MB -231 and HUVECs cells were incubated with AP-PFH
/PLGA NPs at various concentrations, and then the cytotoxicity was tested using a standard CCK-8 assay. As shown in Figure S3A, AP-PFH/PLGA NPs had no significant effect on the survival of MDA-MB-231 and HUVECs cells even when the concentration of NPs increased to as high as $10.0 \mathrm{mg} / \mathrm{mL}$. The result of routine blood tests and blood biochemical analyses showed no apparent difference compared with 
PBS group (Figure S3B-K). Furthermore, H\&E staining of major organs (heart, liver, spleen, lung and kidney) also showed no noticeable damage in AP-PFH/PLGA group (Figure S3L). These results indicated that the dose of APPFH/PLGA NPs used in our work has favorable biosafety for further utilization.

\section{Evaluation of Targeting Ability of AP-PFH/ PLGA NPs to BF}

CLSM and FCM were used to evaluate the binding between $\mathrm{BF}$ and AP-PFH/PLGA NPs in vitro. As shown in Figure 3A, only when AP exists, BF (FITC-labeled BF presents green fluorescence) can connect with PFH/PLGA NPs (DiI-labeled
A
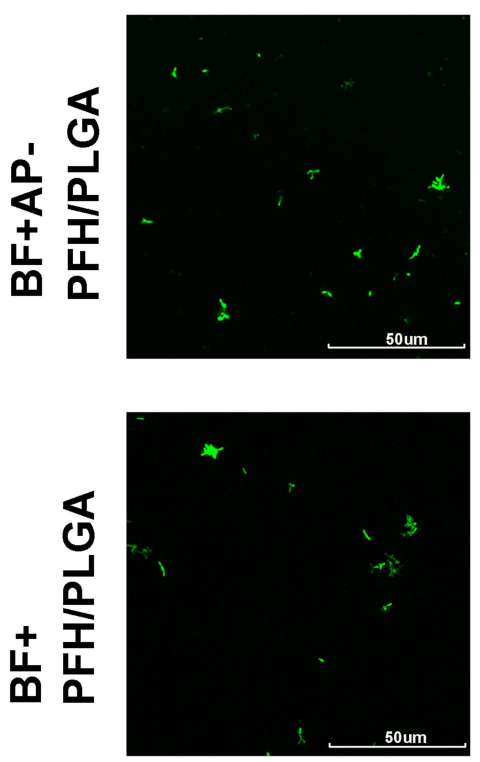

Dil
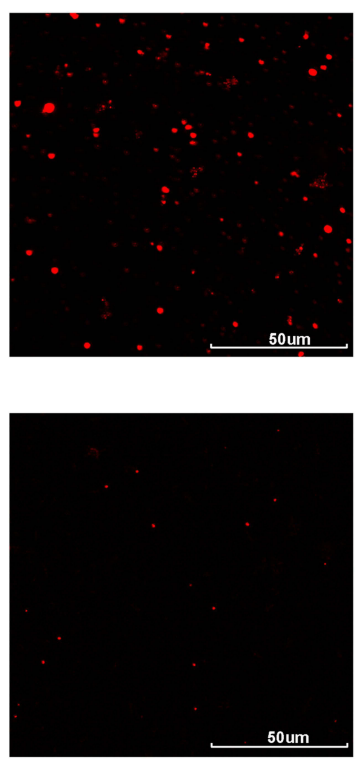

B

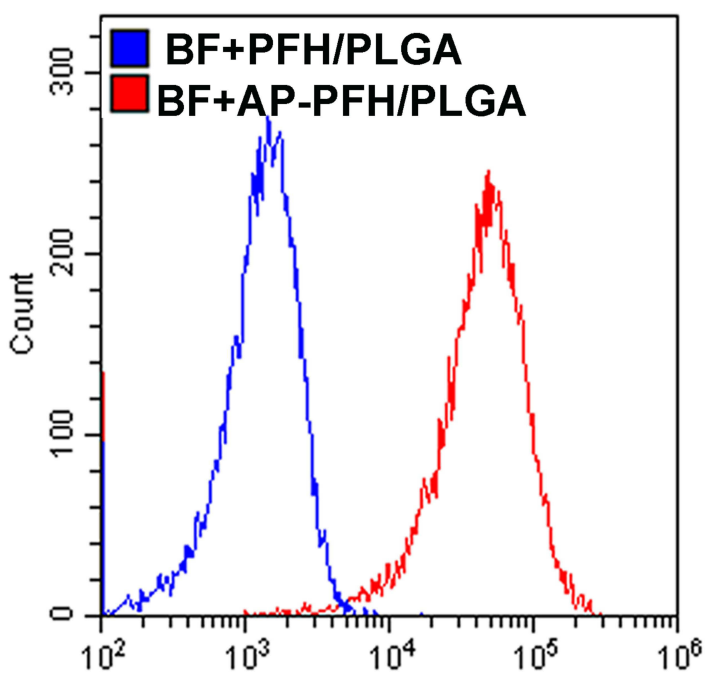

Merge
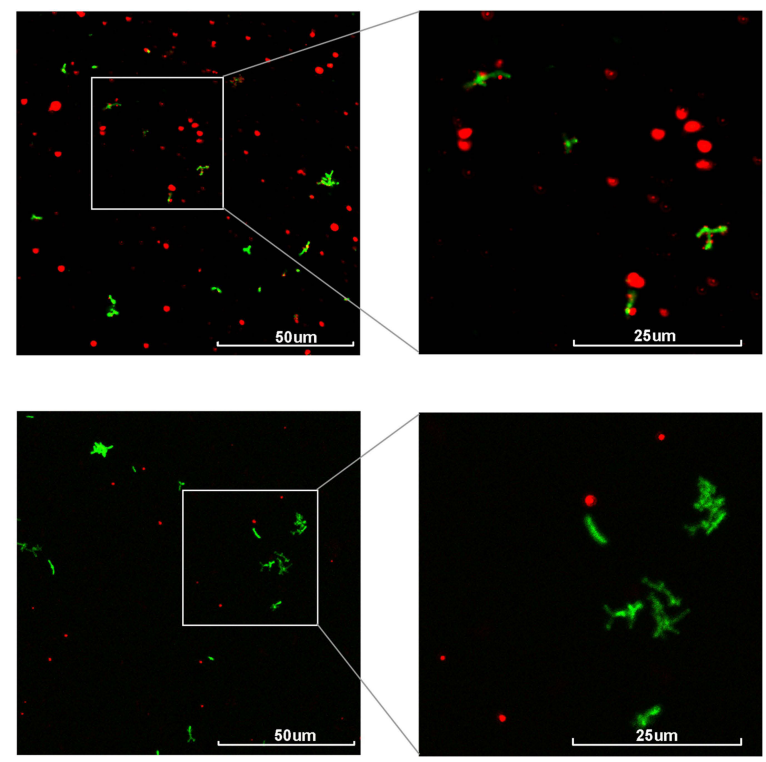

C

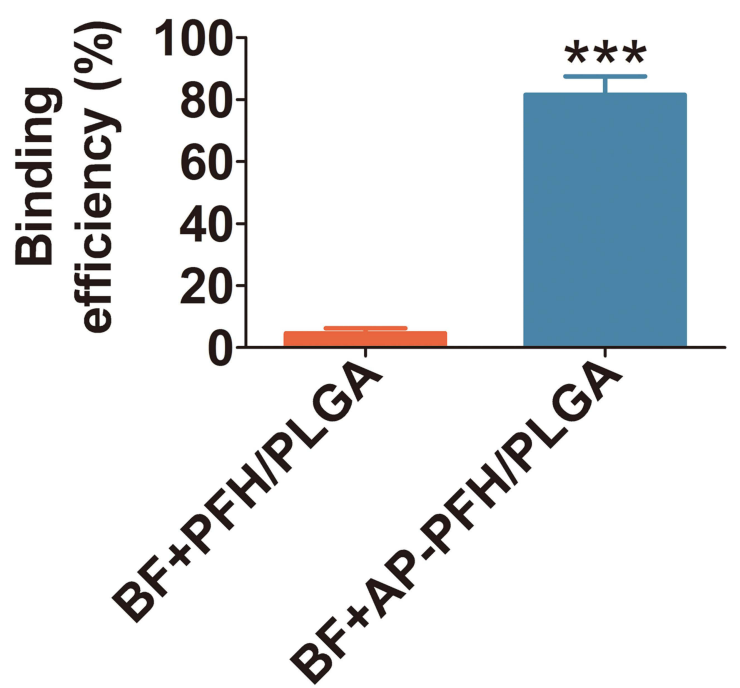

Figure 3 The binding between BF and AP-PFH/PLGA NPs in vitro. (A) CLSM images analysis binding between FITC-labeled BF and Dil-labeled NPs. (B) Flow cytometry analysis binding between BF and Dil-labeled NPs. (C) Binding efficiency between BF and Dil-labeled NPs $(n=3, * * * P<0.00 \mathrm{I})$.

Abbreviations: PFH, perfluorohexane; PLGA, poly(lactic-co-glycolic acid); NPs, nanoparticles; AP, aptamers CCFM64I-5; BF, Bifidobacterium bifidum strain ATCC2952I; FITC, Fluorescein isothiocyanate; Dil, I,I'-dioctadecyl-3,3,3',3'-tetramethylindocarbocyanine perchlorate; CLSM, confocal laser scanning microscope. 
NPs presents red fluorescence). Similarly, the result of FCM showed that (Figure 3B and $\mathrm{C}$ ) the binding efficiency in the $\mathrm{BF}+\mathrm{AP}-\mathrm{PFH} / \mathrm{PLGA}$ group $(81.54 \% \pm 5.98 \%)$ was significantly higher than that of the $\mathrm{BF}+\mathrm{PFH} / \mathrm{PLGA}$ group $(4.64 \%$ $\pm 1.61 \%$ ). These results shown that AP-PFH/PLGA NPs has a high binding ability to BF.

In order to demonstrate whether AP-PFH/PLGA NPs can be guided into tumor tissue based on the tumortargeting ability of BF, fluorescence imaging was performed to detect the presence of NPs in tumor at different time points after injection with DiR-labeled PFH/PLGA NPs or AP-PFH/PLGA NPs and the corresponding fluorescence intensities were quantified. As shown in Figure 4A and B, fluorescence intensity of the $\mathrm{BF}+\mathrm{AP}-\mathrm{PFH} / \mathrm{PLGA}$ group were observed at the tumor site $8 \mathrm{~h}$ after injection, and it increased with time until it reached a peak value $24 \mathrm{~h}$ after injection. The fluorescent signal was still observed in the tumor $48 \mathrm{~h}$ after injection.
A

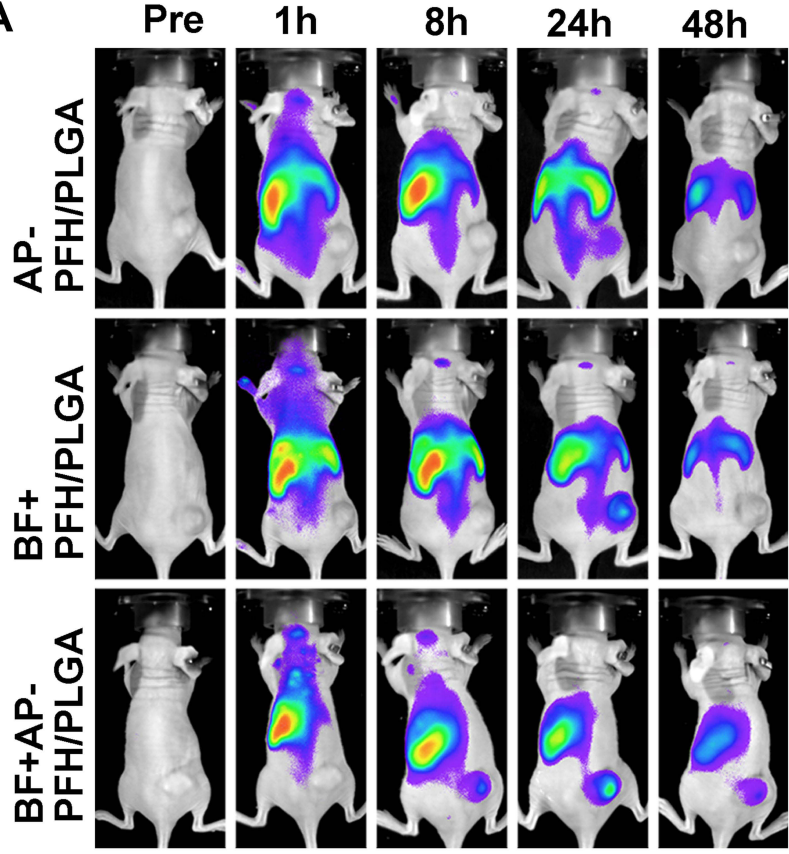

E
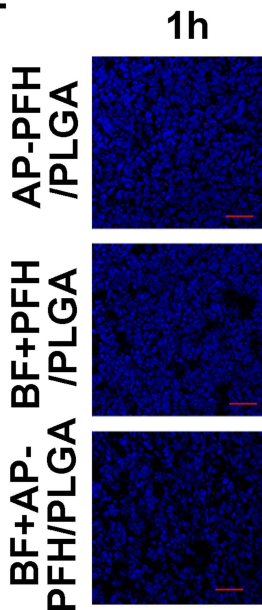

$8 \mathrm{~h}$
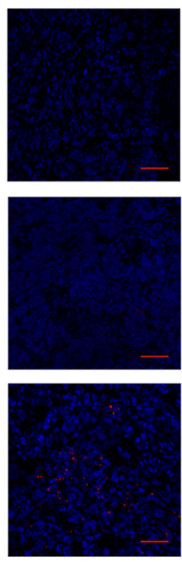

$24 h$
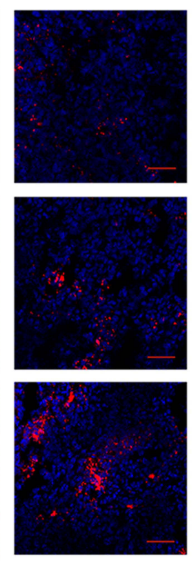

$48 h$
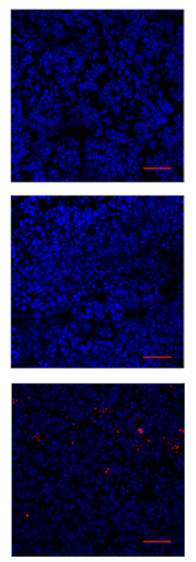

B
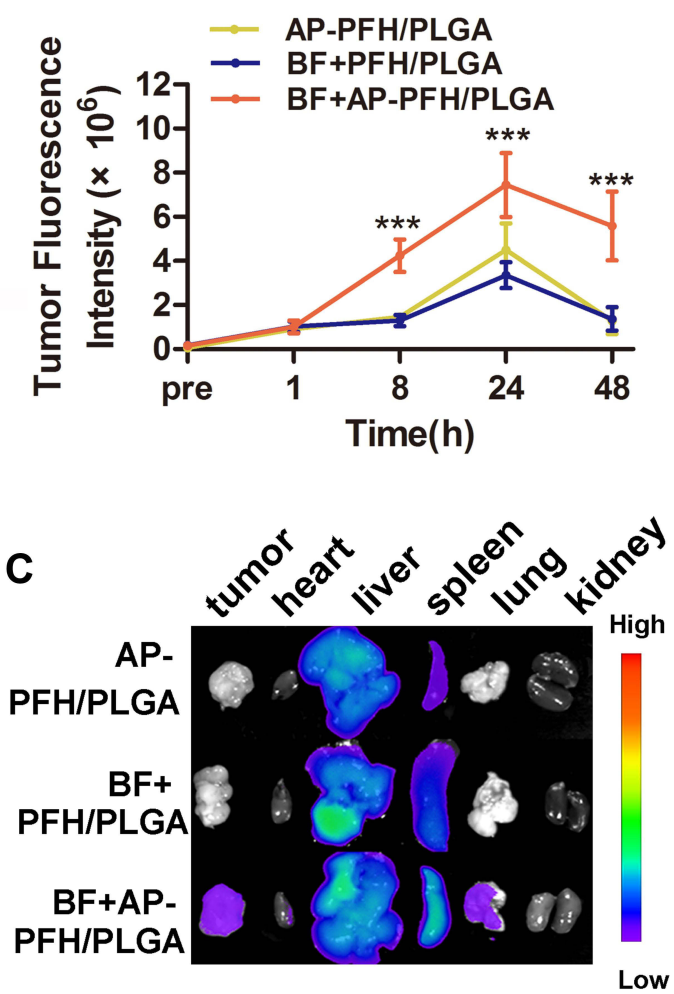

D

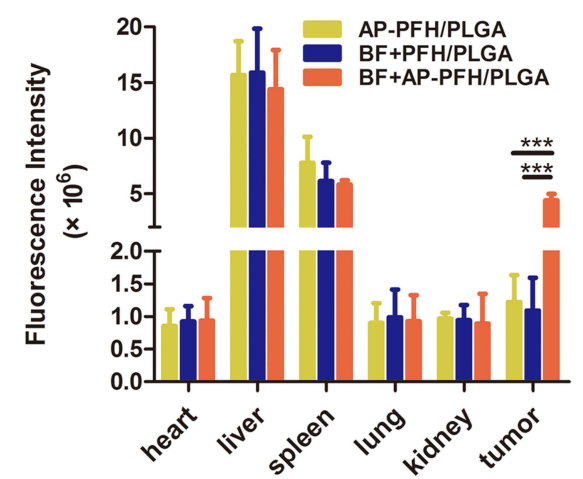

Figure 4 Targeting ability of AP-PFH/PLGA NPs to BF in vivo. (A) Fluorescence imaging and (B) Quantitative fluorescence intensity of tumor tissue before injection and at I h, 8 h, 24 h, 48 h after injection NPs $(n=3, * * *<<0.001)$. (C) Fluorescence imaging and (D) Quantitative analysis of fluorescence intensity of tumor and major organs excised from mice at $48 \mathrm{~h}$ after injection of NPs $(n=3$, *** $<0.00 \mathrm{I})$. (E) Ultrathin section of tumor tissues at I h, $8 \mathrm{~h}, 24 \mathrm{~h}, 48 \mathrm{~h}$ after injection of NPs detected by CLSM. The scale bar is $50 \mu \mathrm{m}$.

Abbreviations: PFH, perfluorohexane; PLGA, poly(lactic-co-glycolic acid); NPs, nanoparticles; AP, aptamers CCFM64I-5; BF, Bifidobacterium bifidum strain ATCC2952I; CLSM, confocal laser scanning microscope. 
Although in the other two groups, fluorescence was observed at the tumor site $24 \mathrm{~h}$ after injection, no strong fluorescence signal was observed in the tumor $48 \mathrm{~h}$ after injection. Moreover, the fluorescence intensity of the BF + AP-PFH/PLGA group was stronger than that of the other two groups at $8 \mathrm{~h}, 24 \mathrm{~h}$ and $48 \mathrm{~h}$ after injection. Next, the major organs and tumors were harvested $48 \mathrm{~h}$ after injection for in vitro fluorescence imaging and the corresponding fluorescence intensities were quantified. Similarly, the results showed that tumor in $\mathrm{BF}+\mathrm{AP}-\mathrm{PFH} / \mathrm{PLGA}$ group retained more of the fluorescent signals compared with AP-PFH/PLGA group and BF+PFH/PLGA group (Figure $4 \mathrm{C}$ and D). We also found that all groups showed abundant fluorescence intensities in the liver and spleen (Figure 4C and D), which can be attributed to the phagocytosis of the reticuloendothelial system. ${ }^{30}$ Consistently, ultrathin tumor sections (Figure 4E) showed that tumor in $\mathrm{BF}+\mathrm{AP}-\mathrm{PFH} / \mathrm{PLGA}$ group contained more NPs $8 \mathrm{~h}, 24$ $\mathrm{h}$ and $48 \mathrm{~h}$ after injection, compared with AP-PFH/PLGA group and $\mathrm{BF}+\mathrm{PFH} / \mathrm{PLGA}$ group.

These results indicate that NPs in all groups can be delivered to solid tumors through blood circulation. However, only when BF is present in tumor, the retention of AP-PFH/PLGA NPs in the tumor is the largest and the retention time is longer. Therefore, a BF-mediated strategy to deliver AP-PFH/PLGA NPs by aptamer-directed approach into solid tumors based on BF is a highly efficient delivery strategy. This lays a foundation for enhancing the treatment efficiency of HIFU.

\section{Evaluation of Synergistic Effect of AP-PFH/PLGA NPs for BF-Mediated HIFU Ablation}

In our preliminary experiment, we have demonstrated that $\mathrm{BF}$ can be colonized in tumor tissues, and the retention of AP-PFH/PLGA NPs in the tumor is the largest and the retention time is longer when $\mathrm{BF}$ is present in tumor. Therefore, a BF-mediated strategy to deliver AP-PFH /PLGA NPs by aptamer-directed approach into tumor based on BF can be used to enhance HIFU ablation. Next, we evaluate the synergistic effect of AP-PFH /PLGA NPs for BF-mediated HIFU ablation. Based on the imaging results, we chose $24 \mathrm{~h}$ after NPs injection as the time point for HIFU ablation. As shown in Figure 5A, varying degrees of ultrasound grayscale changes were observed after ablation in all groups. However, the ultrasound grayscale changes of $\mathrm{BF}+\mathrm{AP}-\mathrm{PFH} / \mathrm{PLGA}$ group was the most obvious and significantly differed from that of other groups (Figure 5B). Next, tumors were harvested $24 \mathrm{~h}$ after HIFU ablation. Then, TTC staining was performed to evaluate the size of coagulation necrosis. The normal tissue appeared as red, and the coagulation necrosis area was not stained and appeared as white (Figure 5C). After HIFU ablation, coagulation necrosis was observed in all groups, however the volume of coagulation necrosis was highest in $\mathrm{BF}+\mathrm{AP}-\mathrm{PFH} / \mathrm{PLGA}$ group compared with that of the other groups (Figure 5D). By further microscopic examination after H\&E staining (Figure 6A), a clear boundary between ablated area and non-ablated area can be observed in all groups. More importantly, BF+AP-PFH/PLGA group could cause large-scale tumor necrosis leaving a mass of substance stained evenly red, compared with other groups. During HIFU ablation, an ultrasonic probe focuses a beam of external high energy on tumor tissue in vivo, which generates a thermal effect and induces coagulative necrosis by transiently raising the tissue temperature to exceed $60^{\circ} \mathrm{C}$. The efficacy of HIFU ablation is low when the focused ultrasound reaches the deep-seated organs due to the attenuation of the ultrasound energy along the ultrasound pathway, which often needs higher ultrasound power and longer ablation duration. The simple increase of ultrasound energy and ablation duration will cause undesired heating in tissues and will lead to unknown adverse events to normal tissue. The introduction of micro- or nanoparticles could change the acoustic environment of tissues, thus enhancing the acoustic energy deposition to improve the therapeutic efficiency of HIFU. ${ }^{5,10}$ This was also confirmed in our study. The results determined that BF-mediated strategy to deliver AP-PFH/PLGA NPs by aptamer-directed approach based on BF can significantly enhance the effect of HIFU ablation due to the AP-PFH/PLGA NPs being able to pass through the vascular endothelia gap and to be retained in the tumor tissue for a long time.

To further observe the effects centered on the cells surrounding tissues over a time course after HIFU ablation, pathological examinations were undertaken. Apoptosis, programmed cell death, is a normal physiologic process maintaining tissue homeostasis and can be induced by heat, cold, ultraviolet radiation and other stimuli. ${ }^{42,43}$ Therefore, we evaluated whether the apoptosis of surrounding tissue of ablated area was influenced after HIFU ablation in different groups by TUNEL assay. TUNEL assay showed that each group had different 


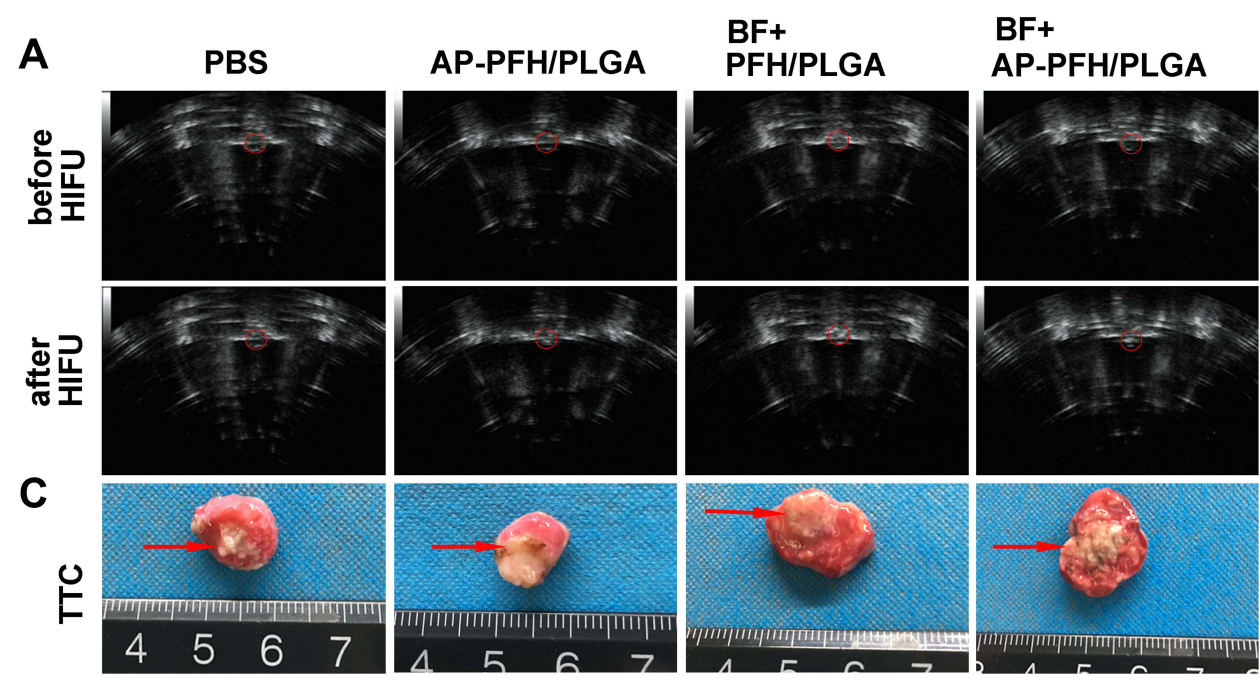

B

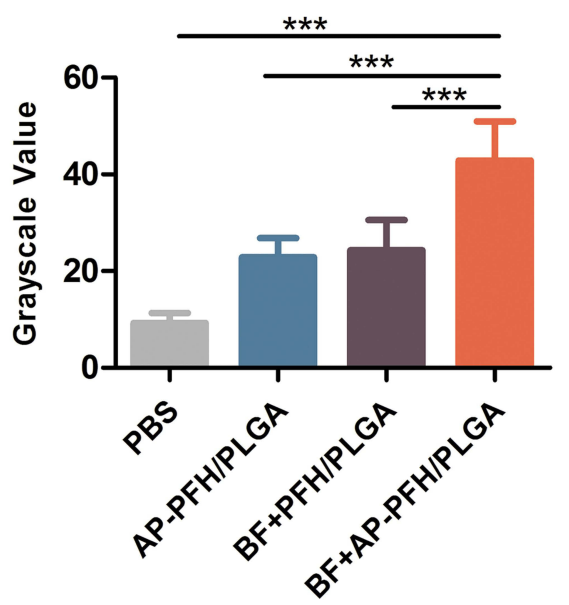

D

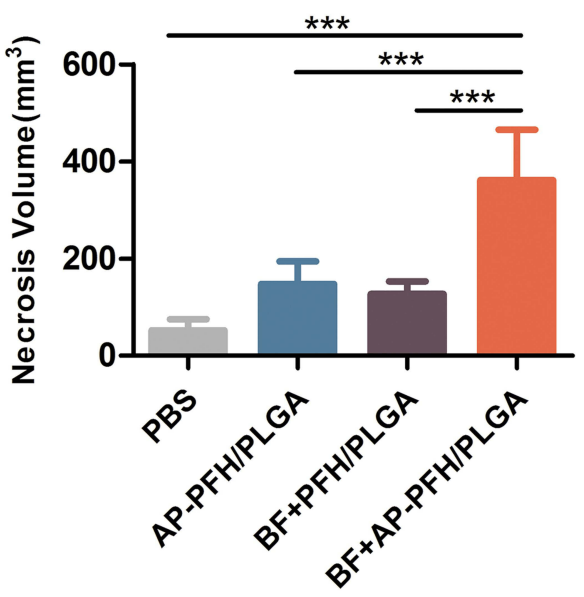

Figure 5 Evaluation of synergistic effect of AP-PFH/PLGA NPs for BF-mediated HIFU ablation in vivo. (A) ultrasonic grayscale changes of tumor tissues (red circle) before and after HIFU ablation and (B) quantitative analysis of grayscale value $(n=10$, ***P $<0.001)$. (C) Coagulation necrosis (red arrow) of tumors by TTC staining after HIFU ablation and (D) quantitative analysis of necrosis volume $(\mathrm{n}=5, * * * \mathrm{P}<0.00 \mathrm{I})$.

Abbreviations: PFH, perfluorohexane; PLGA, poly(lactic-co-glycolic acid); NPs, nanoparticles; AP, aptamers CCFM64I-5; BF, Bifidobacterium bifidum strain ATCC2952I; HIFU, high intensity focused ultrasound.

extents of apoptosis (Figure 6B). The apoptotic index (AI) of tumor cells in the BF+AP-PFH/PLGA group was significantly higher than that of any other groups (Figure S4). Research suggests that heat stress during HIFU exposure enhanced by microbubbles can be related to more apoptotic events. ${ }^{44}$ More apoptosis in the surrounding areas might enlarge the area damaged by HIFU ablation, which might be useful for inhibiting tumor growth after HIFU ablation. Therefore, BF-mediated HIFU ablation can not only improve the ablation efficiency of HIFU, but also can increase the apoptosis of the surrounding tissues of the ablation area. These lay a foundation for HIFU highly efficient treatment of tumors.

\section{In vivo Anti-Tumor Effect of BF-Mediated HIFU Ablation}

The effects of HIFU ablation on tumors were enhanced by AP-PFH/PLGA NPs combined with BF, which may be beneficial in the clinical application of HIFU therapy. To further investigate the potential of the use of BFmediated HIFU ablation for the non-invasive treatment of tumor, we evaluated the therapeutic efficacy of this strategy in vivo. Docetaxel is a potential anti-tumor drug with a broad anti-tumor spectrum and it has shown antitumor effects in breast cancer, non-small cell lung cancer and prostate cancer. ${ }^{35,36,45,46}$ This study used docetaxel as a positive control method to treat breast cancer, and 

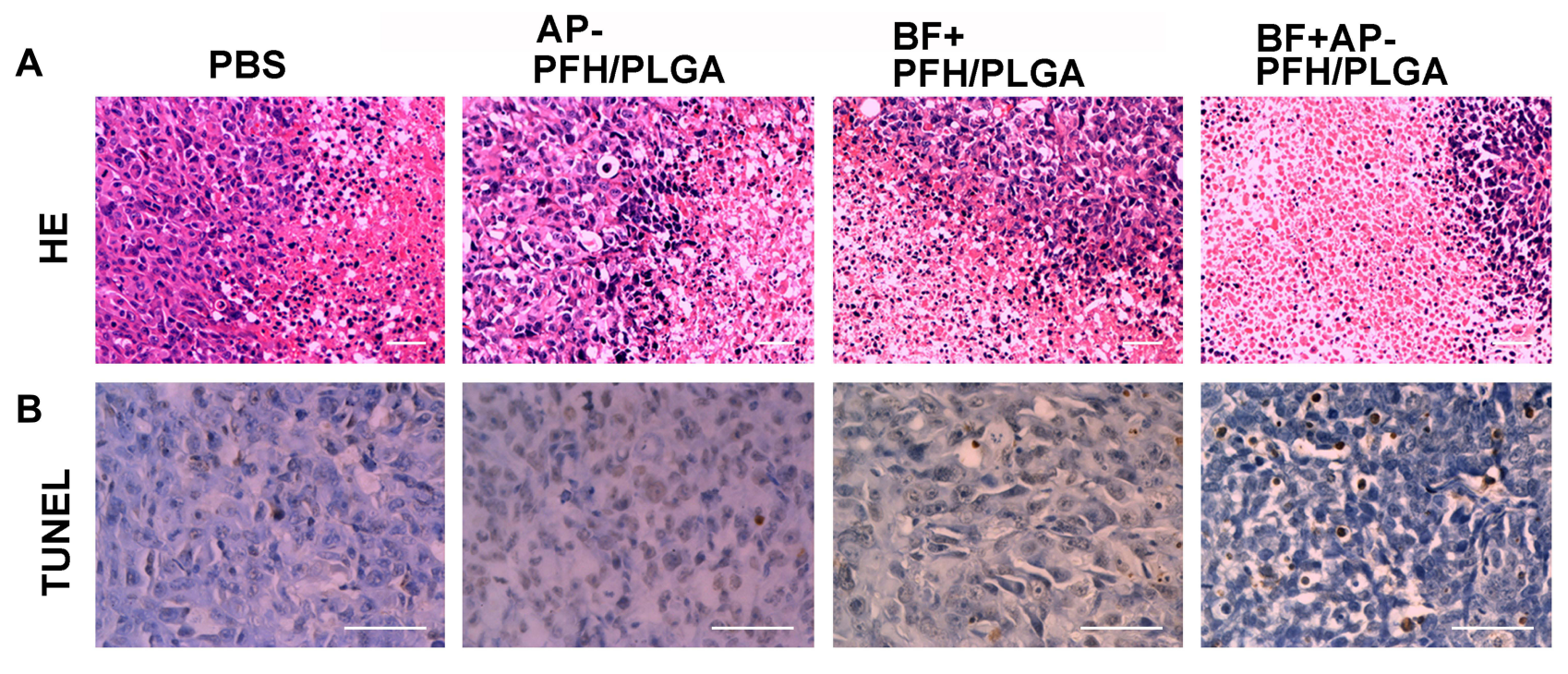

Figure 6 (A) H\&E staining and (B) TUNEL assay in tumor tissue in different groups after HIFU ablation (H\&E: $\times 200$ magnification, TUNEL assay: $\times 400$ magnification). All the scale bars are $50 \mu \mathrm{m}$.

Abbreviations: HIFU, high intensity focused ultrasound; H\&E, hematoxylin and eosin staining; TUNEL, Terminal-deoxynucleotidyl Transferase Mediated Nick End Labeling.

then the efficacy of BF-mediated HIFU ablation technology was assessed in the treatment of breast cancer to demonstrated the potential utility of BF-mediated HIFU ablation.

Overall survival and tumor growth inhibition were used as metrics to determine efficacy. As shown in the Figure 7A and D, both docetaxel group and HIFU+BF + AP-PFH/PLGA group (BF-mediated HIFU ablation) can inhibit the growth of tumors, but the BF-mediated HIFU ablation shows a higher inhibitory effect. We also found that survival times of mice treated with docetaxel (37 days) or saline (28 days) was significantly shorter than those of mice treated with BFmediated HIFU ablation (60 days) (Figure 7B). After BF-mediated HIFU ablation treatment, the weight fluctuations of nude mice bearing MDA-MB-231 xenografts were negligible (Figure 7C). In addition, H\&E staining was performed on the main organs of the mice, including the heart, liver, spleen, lung and kidney after BF-mediated ablation. As shown in Figure

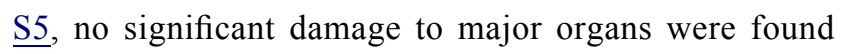
during the treatment period.

These results show that BF-mediated HIFU ablation can significantly inhibit tumor growth, prolong the survival period of mice, and has no significant effect on normal tissues. Overall, BF-mediated HIFU ablation is a new promising therapeutic method for non-invasive tumor ablation.

\section{Conclusion}

This is the first study to report a BF-mediated strategy to deliver AP-PFH/PLGA NPs by aptamer-directed approach into solid tumor based on the tumor-targeting ability of BF to improve the efficiency of HIFU. In summary, we have treated breast cancer more efficiently by taking the novel strategy of BF-mediated HIFU ablation. Specifically, we successfully synthesized AP-PFH/PLGA NPs using double emulsion method and carbodiimide method. The elaborately designed AP-PFH/PLGA NPs can target BF colonized in tumor by receptor-ligand interaction to achieve high tumor accumulation, which can significantly enhance HIFU therapeutic efficiency. Compared with the typical cancer-treatment protocols, such as chemotherapy, BF-mediated HIFU ablation can significantly inhibit tumor growth and prolong the median survival time. Therefore, this novel strategy shows great potential in tumor treatment. Because bifidobacteria target the hypoxic area of the tumor instead of targeting specific proteins or surface antigens overexpressed on cancer cells, ${ }^{21-24}$ theoretically speaking, regardless of the tumor type, when the tumor has a hypoxic area, bifidobacteria can target tumors by virtue of their preference for the hypoxia in the tumor cores. Bifidobacteria can effectively be used in treatment of different tumors. Therefore, BF-mediated HIFU ablation may also be used in the future for a variety of other cancers, including liver cancer, melanoma, head and neck tumors, etc. What needs to be pointed out is that the main limitation of BF-mediated HIFU ablation strategy involves 

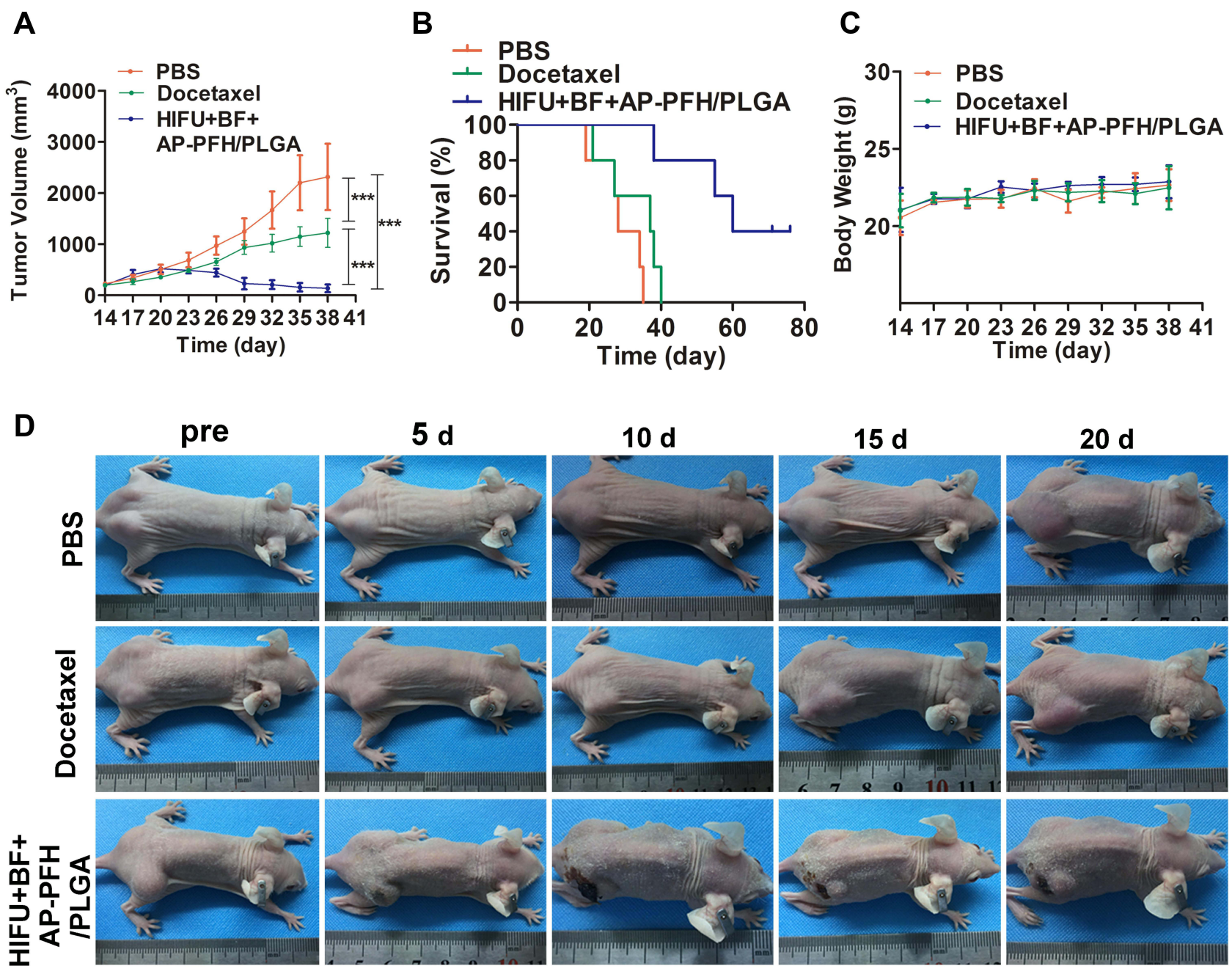

Figure 7 In vivo anti-tumor effect evaluation. (A) Tumor growth inhibition, (B) survival, (C) body weights and (D) photographs after different treatment in mice bearing MDA-MB-23I xenografts $(n=5, * * * P<0.00 I)$

the size of tumor. Research shows that when the tumor is small, the existing vasculature system is able to supply sufficient oxygen and nutrients to the tumor cells until the solid tumors grow to a size of about $1-2 \mathrm{~mm} .{ }^{47}$ For this reason, BF-mediated HIFU ablation may not be a highly efficient strategy for treating tumors with a size of below $1-2 \mathrm{~mm}$. To provides more effective tumor treatment, BF-mediated HIFU ablation strategy could be further optimized by tuning the doses and treatment times as well as incorporating immunotherapy in the future research. In short, our BF-mediated HIFU ablation therapy may provide a new way to treat cancer and also provides a versatile approach for the accumulation enhancement of synergistic agents for HIFU cancer surgery.

\section{Disclosure}

The authors report no conflicts of interest in this work.

\section{References}

1. Kennedy JE. High-intensity focused ultrasound in the treatment of solid tumours. Nat Rev Cancer. 2005;5(4):321-327. doi:10.1038/ nrc1591

2. Napoli A, Alfieri G, Scipione R, et al. High-intensity focused ultrasound for prostate cancer. Expert Rev Med Devices. 2020;17 (5):427-433. doi:10.1080/17434440.2020.1755258

3. Eranki A, Srinivasan P, Ries M, et al. High-intensity focused ultrasound (HIFU) triggers immune sensitization of refractory murine neuroblastoma to checkpoint inhibitor therapy. Clin Cancer Res. 2020;26(5):1152-1161. doi:10.1158/1078-0432.CCR-19-1604

4. Al-Bataineh O, Jenne J, Huber P. Clinical and future applications of high intensity focused ultrasound in cancer. Cancer Treat Rev. 2012;38 (5):346-353. doi:10.1016/j.ctrv.2011.08.004

5. Chen Y, Chen H, Shi J. Nanobiotechnology promotes noninvasive high-intensity focused ultrasound cancer surgery. Adv Healthc Mater. 2015;4(1):158-165. doi:10.1002/adhm.201400127

6. Zhang $\mathrm{N}$, Cai $\mathrm{X}$, Gao $\mathrm{W}$, et al. A multifunctional theranostic nanoagent for dual-mode image-guided HIFU/chemo- synergistic cancer therapy. Theranostics. 2016;6(3):404-417. doi:10.7150/ thno. 13478 
7. You Y, Wang Z, Ran H, et al. Nanoparticle-enhanced synergistic HIFU ablation and transarterial chemoembolization for efficient cancer therapy. Nanoscale. 2016;8(7):4324 4339. doi:10.1039/C5NR08292G

8. He W, Wang W, Zhou P, et al. Enhanced ablation of high intensity focused ultrasound with microbubbles: an experimental study on rabbit hepatic VX2 tumors. Cardiovasc Intervent Radiol. 2011;34 (5):1050-1057. doi:10.1007/s00270-010-0052-3

9. Chung DJ, Cho SH, Lee JM, Hahn ST. Effect of microbubble contrast agent during high intensity focused ultrasound ablation on rabbit liver in vivo. Eur $J$ Radiol. 2012;81(4):e519-e523. doi:10.1016/j. ejrad.2011.06.002

10. Zhou Y, Wang Z, Chen Y, et al. Microbubbles from gas-generating perfluorohexane nanoemulsions for targeted temperature-sensitive ultrasonography and synergistic HIFU ablation of tumors. Adv Mater. 2013;25(30):4123-4130. doi:10.1002/adma.201301655

11. Tang H, Guo Y, Peng L, et al. In vivo targeted, responsive, and synergistic cancer nanotheranostics by magnetic resonance imaging-guided synergistic high-intensity focused ultrasound ablation and chemotherapy. ACS Appl Mater Interfaces. 2018;10 (18):15428-15441. doi:10.1021/acsami.8b01967

12. Kim KT, Lee JY, Kim DD, Yoon IS, Cho HJ. Recent progress in the development of poly(lactic-co-glycolic acid)-based nanostructures for cancer imaging and therapy. Pharmaceutics. 2019;11(6):280. doi:10.3390/pharmaceutics11060280

13. Mir M, Ahmed N, Rehman AU. Recent applications of PLGA based nanostructures in drug delivery. Colloids Surf B Biointerfaces. 2017;159:217-231. doi:10.1016/j.colsurfb.2017.07.038

14. Wang X, Chen H, Chen Y, et al. Perfluorohexane-encapsulated mesoporous silica nanocapsules as enhancement agents for highly efficient high intensity focused ultrasound (HIFU). Adv Mater. 2012;24 (6):785-791. doi:10.1002/adma.201104033

15. Siddique S, Chow JCL. Application of nanomaterials in biomedical imaging and cancer therapy. Nanomaterials. 2020;10(9):1700. doi:10.3390/nano10091700

16. Moore JA, Chow J. Recent progress and applications of gold nanotechnology in medical biophysics using artificial intelligence and mathematical modeling. Nano Ex. 2021;2:022001. doi:10.1088/ 2632-959X/abddd3

17. Siddique S, Chow JCL. Gold nanoparticles for drug delivery and cancer therapy. Appl Sci. 2020;10:3824. doi:10.3390/app10113824

18. He J, Li C, Ding L, et al. Tumor targeting strategies of smart fluorescent nanoparticles and their applications in cancer diagnosis and treatment. Adv Mater. 2019;31(40):e1902409. doi:10.1002/adma.201902409

19. Marusyk A, Polyak K. Tumor heterogeneity: causes and consequences. Biochim Biophys Acta. 2010;1805(1):105-117. doi:10.1016/j.bbcan.2009.11.002

20. McGranahan N, Swanton C. Clonal heterogeneity and tumor evolution: past, present, and the future. Cell. 2017;168(4):613-628. doi:10.1016/j.cell.2017.01.018

21. Kimura NT, Taniguchi S, Aoki K, Baba T. Selective localization and growth of Bifidobacterium bifidum in mouse tumors following intravenous administration. Cancer Res. 1980;40(6):2061-2068.

22. Li X, Fu GF, Fan YR, et al. Bifidobacterium adolescentis as a delivery system of endostatin for cancer gene therapy: selective inhibitor of angiogenesis and hypoxic tumor growth. Cancer Gene Ther. 2003;10(2):105-111. doi:10.1038/sj.cgt.7700530

23. Luo $\mathrm{CH}$, Huang $\mathrm{CT}$, $\mathrm{Su} \mathrm{CH}$, Yeh CS. Bacteria-mediated hypoxia-specific delivery of nanoparticles for tumors imaging and therapy. Nano Lett. 2016;16(6):3493-3499. doi:10.1021/acs. nanolett.6b00262

24. Yazawa K, Fujimori M, Amano J, Kano Y, Taniguchi S. Bifidobacterium longum as a delivery system for cancer gene therapy: selective localization and growth in hypoxic tumors. Cancer Gene Ther. 2000;7(2):269-274. doi:10.1038/sj.cgt.7700122
25. Zhu G, Chen X. Aptamer-based targeted therapy. Adv Drug Deliv Rev. 2018;134:65-78. doi:10.1016/j.addr.2018.08.005

26. Kaur H. Recent developments in cell-SELEX technology for aptamer selection. Biochim Biophys Acta Gen Subj. 2018;1862 (10):2323-2329. doi:10.1016/j.bbagen.2018.07.029

27. Barbas AS, Mi J, Clary BM, White RR. Aptamer applications for targeted cancer therapy. Future Oncol. 2010;6(7):1117-1126. doi:10.2217/fon.10.67

28. Kinghorn AB, Fraser LA, Lang S, Shiu SCC, Tanner JA. Aptamer bioinformatics. Int J Mol Sci. 2017;18(12):2516. doi:10.3390/ ijms 18122516

29. Hu L, Wang L, Lu W, Zhao J, Zhang H, Chen W. Selection, characterization and interaction studies of a DNA aptamer for the detection of Bifidobacterium bifidum. Int J Mol Sci. 2017;18(5):883. doi:10.3390/ijms18050883

30. Huang J, Liu F, Han X, et al. Nanosonosensitizers for highly efficient sonodynamic cancer theranostics. Theranostics. 2018;8 (22):6178-6194. doi:10.7150/thno.29569

31. Zhang X, Zheng Y, Wang Z, et al. Methotrexate-loaded PLGA nanobubbles for ultrasound imaging and synergistic targeted therapy of residual tumor during HIFU ablation. Biomaterials. 2014;35 (19):5148-5161. doi:10.1016/j.biomaterials.2014.02.036

32. Farokhzad OC, Jon S, Khademhosseini A, Tran TN, Lavan DA, Langer R. Nanoparticle-aptamer bioconjugates: a new approach for targeting prostate cancer cells. Cancer Res. 2004;64(21):7668-7672. doi:10.1158/0008-5472.CAN-04-2550

33. Guo J, Gao X, Su L, et al. Aptamer-functionalized PEG-PLGA nanoparticles for enhanced anti-glioma drug delivery. Biomaterials. 2011;32(31):8010-8020. doi:10.1016/j.biomaterials.2011.07.004

34. Handké N, Ficheux D, Rollet M, et al. Lysine-tagged peptide coupling onto polylactide nanoparticles coated with activated ester-based amphiphilic copolymer: a route to highly peptide-functionalized biodegradable carriers. Colloids Surf B Biointerfaces. 2013;103:298-303. doi:10.1016/j.colsurfb.2012.10.032

35. Zeng J, Liu W, Fan YZ, He DL, Li L. PrLZ increases prostate cancer docetaxel resistance by inhibiting LKB1/AMPK-mediated autophagy. Theranostics. 2018;8(1):109-123. doi:10.7150/thno.20356

36. Desai NP, Trieu V, Hwang LY, Wu R, Soon-Shiong P, Gradishar WJ. Improved effectiveness of nanoparticle albumin-bound (nab) paclitaxel versus polysorbate-based docetaxel in multiple xenografts as a function of HER2 and SPARC status. Anticancer Drugs. 2008;19 (9):899-909. doi:10.1097/CAD.0b013e32830f9046

37. Kim KS, Youn YS, Bae YH. Immune-triggered cancer treatment by intestinal lymphatic delivery of docetaxel-loaded nanoparticle. J Control Release. 2019;311-312:85-95. doi:10.1016/j. jconrel.2019.08.027

38. Bowerman CJ, Byrne JD, Chu KS, et al. Docetaxel-loaded PLGA nanoparticles improve efficacy in taxane-resistant triple-negative breast cancer. Nano Lett. 2017;17(1):242-248. doi:10.1021/acs. nanolett.6b03971

39. Michieli P. Hypoxia, angiogenesis and cancer therapy: to breathe or not to breathe? Cell Cycle. 2009;8(20):3291-3296. doi:10.4161/ cc.8.20.9741

40. Dachs GU, Patterson AV, Firth JD, et al. Targeting gene expression to hypoxic tumor cells. Nat Med. 1997;3(5):515-520. doi:10.1038/ nm0597-515

41. Wu M, Wang Y, Wang Y, et al. Paclitaxel-loaded and A10-3.2 aptamer-targeted poly (lactide-co-glycolic acid) nanobubbles for ultrasound imaging and therapy of prostate cancer. Int J Nanomedicine. 2017;12:5313-5330. doi:10.2147/IJN.S136032

42. Saliev T, Feril LB, Nabi G, Melzer A. Targeted manipulation of apoptotic pathways by using high intensity focused ultrasound in cancer treatment. Cancer Lett. 2013;338(2):204-208. doi:10.1016/j. canlet.2013.04.016 
43. Barry MA, Behnke CA, Eastman A. Activation of programmed cell death (apoptosis) by cisplatin, other anticancer drugs, toxins and hyperthermia. Biochem Pharmacol. 1990;40(10):2353-2362. doi:10.1016/0006-2952(90)90733-2

44. Luo W, Zhou X, Zhang J, et al. Analysis of apoptosis and cell proliferation after high intensity-focused ultrasound ablation combined with microbubbles in rabbit livers. Eur $J$ Gastroenterol Hepatol. 2007;19(11):962-968. doi:10.1097/MEG.0b013e3282 cfb6f0

45. Arrieta O, Barrón F, Ramírez-Tirado LA, et al. Efficacy and safety of pembrolizumab plus docetaxel vs docetaxel alone in patients with previously treated advanced non-small cell lung cancer: the PROLUNG phase 2 randomized clinical trial. JAMA Oncol. 2020;6 (6):856-864. doi:10.1001/jamaoncol.2020.0409
46. Barata PC, Sartor AO. Metastatic castration-sensitive prostate cancer: abiraterone, docetaxel, or .... Cancer. 2019;125(11):1777-1788. doi:10.1002/cncr.32039

47. Tee JK, Yip LX, Tan ES, et al. Nanoparticles' interactions with vasculature in diseases. Chem Soc Rev. 2019;48(21):5381-5407. doi:10.1039/C9CS00309F

\section{Publish your work in this journal}

The International Journal of Nanomedicine is an international, peerreviewed journal focusing on the application of nanotechnology in diagnostics, therapeutics, and drug delivery systems throughout the biomedical field. This journal is indexed on PubMed Central, MedLine, CAS, SciSearch ${ }^{\circledR}$, Current Contents ${ }^{\circledR} /$ Clinical Medicine, $^{2}$
Journal Citation Reports/Science Edition, EMBase, Scopus and the Elsevier Bibliographic databases. The manuscript management system is completely online and includes a very quick and fair peer-review system, which is all easy to use. Visit http://www.dovepress.com/ testimonials.php to read real quotes from published authors. 